\title{
Fermat's Last Theorem Proven in One Page
}

\author{
Do Tan $\mathrm{Si}^{1,2}$
}

\author{
${ }^{1}$ HoChiMinh-city Physical Association, Vietnam \\ ${ }^{2}$ ULB and UEM, Belgium \\ Correspondence: Do Tan Si, HoChiMinh-city Physical Association, Vietnam. E-mail: tansi_do@yahoo.com
}

Received: March 2, 2018

Accepted: March 16, 2018

Online Published: May 31, 2018

doi:10.5539/apr.v10n3p18

URL: https://doi.org/10.5539/apr.v10n3p18

\begin{abstract}
We attempt to prove the Fermat's Last Theorem by a simple method consisted in transforming the relation $b^{m}=(a+n)^{m}-a^{m}$ into an equation in $n$ by introduction of a parameter $\omega$ depending in $a, n$ such that $b=n \omega^{m(m-1)}$ then equalizing $b$ in these two relations. Afterward, exploiting the condition that this equation must have only one root so that the coefficients of powers of $n$ must have alternating signs, we arrive to conclude that the equation in $n$ has roots only for $m=1,2$ and no root for $m>2$ thus prove the theorem.
\end{abstract}

Keywords: Fermat's Last Theorem, Pythagorean triples

\section{Introduction}

Fermat's last theorem is a theorem first proposed in 1657 by Fermat in the form of a note scribbled in the margin of his copy of the ancient Greek text Arithmetica by Diophantus (Wikipedia 2018). As everyone knows, the theorem states that the equation $x^{m}+y^{m}=z^{m}$ has no integer solutions for $m>2$ and $x, y, z \neq 0$ but no proof has been given by Fermat himself. Attempts to prove this theorem with more or less success during 338 years are numerous and may be found with comments of eminent mathematicians in literature for example in Wikipedia and plenty of references in it. Finally in 1995 Andrew Wiles published a convincing proof of this famous theorem and received accordingly the prestigious Abel price. The proof is more than 100 pages long.

Regarding the proof given by Wiles (1995), many scientists think that it would not be the proof predicated by Fermat, if it exists. From this conviction it has a meaningn to look for another proof than that of Wiles.

After reflexions suggested by our research for obtaining Pythagorean triples not by the constatation $\left(m^{2}-n^{2}\right)^{2}+4 m^{2} n^{2}=\left(m^{2}+n^{2}\right)^{2}$ but by the fructful reasoning about how to transform a rectangle with integer lenghts into a square with integer lenght having the same superficies, we are generalizing the method to prove the Fermat's last theorem.

The method consists in searching from the relation $a^{m}+b^{m}=(a+n)^{m}$ for an equation on $n$ which must have only one root then show that the latter condition is fulfilled only for $m \leq 2$, as explained in the following chapters.

\section{Obtaining Pythagorean triples}

Consider the following relation between three integers $a, b, n$ representing the measures of some physical quantity such as lenght

$$
a^{2}+b^{2}=(a+n)^{2}
$$

and the problem of obtaining simultaneously three integers $(a, b, a+n)$ called Pythagorean triple (Wikipedia 2018) from it.

From (1) we have

$$
b^{2}=n^{2}+2 a n=n(n+2 a)
$$

In order to transform the second member of (2) into square of a linear term let us search for a scalar $\omega \in Q$ such that

$$
b^{2}=n^{2}+2 a n=n(n+2 a)=\omega n \frac{n+2 a}{\omega}
$$

and

$$
\omega n=\frac{n+2 a}{\omega}
$$

With these conditions we get the relations 


$$
\begin{gathered}
\left(\omega^{2}-1\right) n=2 a \\
b=n \omega
\end{gathered}
$$

From (5) (6) we see that given any rational number $\omega>1$ we put

$$
\omega^{2}-1=\frac{p}{q}
$$

and obtain the smallest values of $(a, b, c)$

$$
(a, b, c)=\left(\frac{p}{2}, q \omega, \frac{p}{2}+q\right) \text { or }(a, b, c)=(p, 2 q \omega, p+2 q) \text { if } p \text { odd }
$$

For example

$$
\begin{gathered}
\omega=17 / 7 \\
\omega^{2}-1=\frac{289-49}{49}=\frac{240}{49} \Rightarrow a=120, n=49, b=119
\end{gathered}
$$

\section{Obtaining Fermat's last Theorem}

Consider the following relation between three positive integers $a, b, n$ representing the measures of some physical quantity such as lenght

$$
a^{m}+b^{m}=(a+n)^{m}
$$

and the problem of obtaining simultaneously $a, b, n$ from it.

Eq. (9) implies $n<b, n$ is unique once $a, b$ known, and

$$
b^{m}=n^{m}+\left(\begin{array}{c}
m \\
1
\end{array}\right) a n^{m-1}+\left(\begin{array}{c}
m \\
2
\end{array}\right) a^{2} n^{m-2}+\ldots+\left(\begin{array}{c}
m \\
m-1
\end{array}\right) a^{m-1} n
$$

In order to transform the second member of (10) into power of order $m$ of only one term let us search for a scalar parameter $\omega$ such that

$$
b^{m}=\left(\omega^{m-1} n\right)\left(\frac{1}{\omega}\left(\frac{b^{m}}{n}\right)^{\frac{1}{m-1}}\right)^{m-1}
$$

and that

$$
\omega^{m-1} n=\frac{1}{\omega}\left(\frac{b^{m}}{n}\right)^{\frac{1}{m-1}}
$$

Eqs (10), (12) lead to the relations

$$
\begin{gathered}
b^{m}=\left(\frac{1}{\omega}\left(\frac{b^{m}}{n}\right)^{\frac{1}{m-1}}\right)^{m} \\
\omega^{m-1}=\frac{b}{n}>1
\end{gathered}
$$

Eqs (10), (14) lead to the equation for obtaining $n$

i.e.

$$
n^{m} \omega^{m(m-1)}=n^{m}+\left(\begin{array}{c}
m \\
1
\end{array}\right) a n^{m-1}+\left(\begin{array}{c}
m \\
2
\end{array}\right) a^{2} n^{m-2}+\ldots+\left(\begin{array}{c}
m \\
m-1
\end{array}\right) a^{m-1} n
$$

$$
\left(1-\omega^{m(m-1)}\right) n^{m-1}+\left(\begin{array}{c}
m \\
1
\end{array}\right) a n^{m-2}+\left(\begin{array}{c}
m \\
2
\end{array}\right) a^{2} n^{m-3}+\ldots+\left(\begin{array}{c}
m \\
m-1
\end{array}\right) a^{m-1}=0
$$

Eq. (15) define $\omega$ in function of $a, n$ so that if nesessary we write also $\omega(a, n)$. 
Discussions:

- Lemma:

If an equation

$$
a(n) b(n)+c(n)=0
$$

has $k$ solutions $n_{1}, n_{2}, \ldots, n_{k}$ then the equation

$$
\begin{gathered}
a\left(n_{l}\right) b(n)+c(n)=0 \\
\text { has } n_{l} \text { as solution. }
\end{gathered}
$$

The proof is evident.

- Let $\omega(a, n)$ be a function verifying the condition (14) and the equation (15). If it is so that (15) has no integer root the Fermat's last theorem would verified. If it is so that (15) has more than one roots there is contradiction with (14). If it is so that (15) has only one root say $n_{l}$ then applying the lemma (16) we may write

$$
\begin{gathered}
\left(1-\omega^{m(m-1)}\left(n_{1}\right)\right) n^{m-1}+\left(\begin{array}{c}
m \\
1
\end{array}\right) a n^{m-2}+\left(\begin{array}{c}
m \\
2
\end{array}\right) a^{2} n^{m-3}+\ldots+\left(\begin{array}{c}
m \\
m-1
\end{array}\right) a^{m-1} \\
=\left(1-\omega^{m(m-1}\left(n_{1}\right)\right)\left(n-n_{1}\right)^{m-1}=0
\end{gathered}
$$

- From (17) we see that the coefficients of $n^{i}$ in the left hand member must alternate in signs.

For $m=1$ we get $0 n=0$ so that any integer is solution of (9)

For $m=2$ we have $\omega>1$ so that $\left(1-\omega^{2}\left(n_{1}\right)\right)<0$. We see then that the equation (17) may have solution. For $m>2$, as the coefficients of the $2^{\text {nd }}$ untill the last terms in the left hand member of (17) are all positive, we see that the said condition is not satisfied so that $n_{l}$ doesn't exist.

This is to say that the equation (9)

$$
a^{m}+b^{m}=(a+n)^{m}
$$

have only solution only for $m=1,2$ and doesn't have solution for $m>2$ as stated the Fermat's last theorem. QED

\section{Remarks amd Conclusion}

When trying to find another way than the old ones for obtaining Pythagorean triples $a^{2}+b^{2}=(a+n)^{2}$ we realize that we firstly have to change the product $n(n+2 a)$ into $\omega n \frac{n+2 a}{\omega}=(\omega n)^{2}$ in order to secondly get the relation $\left(1-\omega^{2}\right) n=2 a$ giving $a, n, b$ for each arbitrary choice of $\omega \in Q$. Generalization for the case $a^{m}+b^{m}=(a+n)^{m}$ is straightforward as shown in (13). This leads to a relation between $\omega, a, n$ where $\omega$ depends somehow in $a, n$. From this relation, for any choice of $\omega(a, n)$ we get an equation in $n$. This equation may have theorically a root $n_{l}$ that we need not calculate. From this root $n_{1}$ which must be unique and by the lemma (16) we get a polynomial equation in $n$. The condition that $n_{l}$ must be unique finally prove the last theorem of Fermat.

\section{Acknowledgement}

The author wishes that if this work is accepted for publication he may receive many "like" from readers, especially from Prof. Paul Van Praag at Brussels and Mons, VPM Vu Duc Dam at Ha Noi, HoChiMinh-city Pdt Nguyen Thien Nhan, BelUnion in Vietnam and Truong Son-UVB in Belgium who always appreciate his love for researches.

\section{References}

Pythagorean triple. (2018). In Wikipedia, the free encyclopedia. Retrieved from https://en.wikipedia.org/wiki/ Pythagorean_triple

Wiles, A. (1995). Modular elliptic curves and Fermat's Last Theorem. Annals of Mathematics, 141(3), 443-451. https://doi.org/10.2307/2118559

Wiles's proof of Fermat's Last Theorem. (2015). In Wikipedia, the free encyclopedia. Retrieved from https://en.wikipedia.org/wiki/Wiles\%27s_proof_of_Fermat\%27s_Last_Theorem

\section{Copyrights}

Copyright for this article is retained by the author(s), with first publication rights granted to the journal.

This is an open-access article distributed under the terms and conditions of the Creative Commons Attribution license (http://creativecommons.org/licenses/by/4.0/). 